\title{
ANÁliSE DA SATISFAÇÃO DOS USUÁRIOS DOS SERVIÇOS DE CLÍNICAS MÉDICA E CIRÚRGICA EM UM HOSPITAL DO SUDOESTE DO PARANÁ ${ }^{1}$
}

\author{
ANALYSIS OF SATISFACTION OF USERS OF SERVICES CLINICAL MEDICAL \\ AND SURGICAL HOSPITAL IN A SOUTHWESTERN PARANÁ
}

\section{ANÁLISIS DE LA SATISFACCIÓN DE LOS CLIENTES Y SERVICIOS CLÍNICOS QUIRÚRGICO MÉDICO EN UN HOSPITAL DE SUROESTE PARANÁ}

\author{
DIEGO CERIOLI \\ Universidade Estadual do Oeste do Paraná \\ diego_cerioli@hotmail.com
}

\author{
GILBERTO FRANCISCO CERETTA \\ Universidade Estadual da Oeste do Paraná \\ gilbertoceretta@gmail.com
}

\author{
ADILSON CARLOS DA ROCHA \\ Universidade Estadual da Oeste do Paraná \\ adilson28@hotmail.com \\ NILSA MARIA GUARDA CANTERLLE \\ Universidade Estadual da Oeste do Paraná \\ nilsacanterle@hotmail.com
}

\begin{abstract}
RESUMO
O presente trabalho foi realizado na instituição hospitalar da Região Sudoeste do Paraná cuja atividade principal consiste na prestação de serviços especializados em saúde, oferecidos por meio do Sistema Único de Saúde (SUS). Este artigo tem como objetivo apresentar a qualidade dos serviços verificada na sua prestação pela referida instituição, a partir do nível de satisfação evidenciada pelos usuários dos serviços das clínicas médica e cirúrgica. A pesquisa possui abordagem qualitativa e quantitativa, foi realizada a partir de uma amostra de 56 pacientes, selecionados por aceitação e conveniência dos pesquisados e utilizou como instrumento de coleta de dados um questionário baseado no modelo SERVPERF. A análise dos dados foi realizada a partir da estatística descritiva, sendo avaliado o nível de satisfação dos pacientes com o serviço prestado pelo hospital. Ao final do estudo observou-se que a instituição apresenta um alto nível de satisfação de seus usuários, indicando que oferece serviços de qualidade elevada. Dentre os elementos do serviço mais bem avaliados estão acessibilidade, ambiente físico, atenção dos funcionários e gentileza do corpo de colaboradores da instituição.
\end{abstract}

PALAVRAS CHAVE: Saúde. Qualidade. Satisfação.

\section{ABSTRACT}

This research was carried out in the hospital from Southwestern Paraná whose main activity is the provision of specialist health services, offered through the Unified Health System (SUS). This article aims to present the quality of service experienced in the provision by that institution, from the level of satisfaction shown by service users from general medical and surgical. The research has qualitative and quantitative approach was conducted from a sample of 56 patients, selected for acceptance and convenience of the students and used as a tool for data collection a questionnaire based on the SERVPERF model. Data analysis was performed from the descriptive statistics were used to evaluate the level of patient satisfaction with the service provided by the hospital. At the end of the study it was observed that the institution has a high level of user satisfaction, indicating that offers high quality services. Among the elements of the service are better evaluated accessibility, physical environment, attention and kindness of the staff corps of the institution.

KEYWORDS: Health Quality. Satisfaction.

\section{RESUMEN}

Este trabajo se llevó a cabo en un hospital de la región del sudoeste de Paraná, cuya actividad principal es la prestación de servicios de salud especializados, ofrecidos a través del Sistema Único de Salud (SUS). Este artículo tiene como objetivo presentar la calidad de servicio con experiencia en la prestación por dicha

\footnotetext{
${ }^{1}$ Submetido em 30 de maio de 2013. Aceito em 10 de outubro de 2013. O artigo foi avaliado segundo o processo de duplo anonimato além de ser avaliado pelo editor. Editores responsáveis: Márcio Augusto Gonçalves e Lucas Maia dos Santos. Reprodução parcial ou total e trabalhos derivativos permitidos com a citação apropriada da fonte.
} 
institución, desde el nivel de satisfacción evidenciado por los usuarios del servicio de clínicas médicas y quirúrgicas. La investigación tiene cualitativo y enfoque cuantitativo se llevó a cabo a partir de una muestra de 56 pacientes seleccionados para la aceptación y la comodidad de los estudiantes y se utilizan como una herramienta para la recopilación de datos, un cuestionario basado en el modelo SERVPERF. El análisis de datos se realizó mediante estadística descriptiva, y se evaluó el nivel de satisfacción del paciente con el servicio prestado por el hospital. Al final del estudio se observó que la institución cuenta con un alto nivel de satisfacción de los usuarios, lo que indica que ofrecen servicios de alta calidad. Entre los elementos del servicio son mejor evaluadas accesibilidad, entorno físico, la atención y la amabilidad del personal del cuerpo de empleados de la institución.

PALABRAS CLAVE: salud de calidad. Satisfacción.

\section{INTRODUÇÃO}

Com a evolução da tecnologia e o crescente aumento da diversidade de produtos e serviços, cada vez mais sofisticados e atraentes, os consumidores se veem cercados por ofertas e vantagens de toda ordem. Neste contexto, as organizações necessitam aumentar seus esforços, gradativamente, na busca pela melhoria da qualidade de seus produtos e serviços, como forma de se diferenciar no mercado e, desta maneira, atrair ou manter seus clientes.

Atender às necessidades e até mesmo superá-las torna-se fundamental, pois a satisfação do cliente passa a ser um fator crucial para a sobrevivência das empresas, principalmente as de serviços, cujos produtos se caracterizam pela intangibilidade. Esta também deve ser uma preocupação das organizações de saúde, que devem oferecer um bom atendimento ao seu cliente, em outros termos - paciente, como forma de mitigar seu sofrimento durante o período de internação, permitindo que sua recuperação seja rápida e o mesmo fique o mínimo de tempo possível afastado de seu lar e do convívio social.

Neste movimento pela melhoria da qualidade, pode ser observado que o sistema público de saúde, apesar de acumular uma defasagem histórica, tanto em sua estrutura física quanto de recursos humanos, têm tido uma evolução significativa nos últimos anos. Essa evolução é corroborada pela aprovação dos serviços de saúde no País que alcançou 86,4\%, conforme apontou a Pesquisa Nacional por Amostra de Domicílios (PNAD), A pesquisa destacou que 26,7 milhões de brasileiros que se consultaram na rede pública ou privada nas duas semanas anteriores ao levantamento, mais de 23 milhões consideraram "muito bom ou bom" o atendimento recebido e $95 \%$ das pessoas foram atendidas na primeira tentativa (IBGE, 2007).

Sendo as instituições que prestam serviços a saúde, que possuem como função primordial, a prestação de assistência à saúde da população e, portanto, possuem grande repercussão social, pode-se considerar como fundamental todo e qualquer esforço que vise aperfeiçoar a qualidade de seus serviços com vistas a excelência na prestação dos serviços.

Neste contexto emerge uma questão problema: Os usuários dos serviços de clinicas médica e cirúrgica prestados pelas organizações de saúde estão satisfeitos com o serviço prestado? Diante desta questão foi desenvolvido um estudo com usuários dos serviços prestados pelas clinicas médica e cirúrgica do Hospital Regional do Sudoeste do Paraná HRS, com o objetivo de verificar a qualidade dos serviços prestados pela organização, por meio do nível de satisfação de seus usuários.

Este artigo está estruturado em seis seções. A primeira corresponde à introdução, a segunda seção trata da gestão de serviços e gestão hospitalar, a terceira contextualiza os modelos SERVQUAL e SERVPERF, bem com a instituição estudada. Logo após, segue o método utilizado e análise e discussão dos resultados. Por fim, apresentam-se as considerações finais para o estudo. 


\section{GESTÃO DE SERVIÇOS}

Os serviços, de acordo com Lovelock e Wright (2001, p. 5), "são atividades econômicas que criam valor e fornecem benefícios para clientes em tempos e lugares específicos, como decorrência da realização de uma mudança desejada no - ou em nome do destinatário do serviço". Lovelock e Wright (2001) citam ainda que, por se tratarem, em grande parte, de insumos e produtos intangíveis, os serviços se tornam de difícil definição.

O setor de serviços, segundo Siqueira (2005), pode ser considerado como uma tendência mundial, de modo que seu crescimento é bem mais elevado do que os demais setores. $\mathrm{O}$ autor ainda menciona que no Brasil, o setor de serviços passou a ser o principal empregador de mão de obra desde a década de 1950, sendo, atualmente, responsável por mais de $60 \%$ da ocupação de mão e obra e cerca de $60 \%$ do Produto Interno Bruto (PIB).

Por conta disso, Corrêa e Caon (2006) consideram o setor de serviços como a parte mais dinâmica da economia. Os autores ainda citam que os principais fatores que levaram os serviços a ter esta ascensão foram a urbanização, as mudanças demográficas e socioeconômicas, o aumento da sofisticação dos consumidores e as mudanças tecnológicas.

Com a evolução tecnológica e uma diversidade de produtos e serviços, cada vez mais sofisticados e atraentes, o consumidor passou a ser mais exigente quanto à qualidade dos produtos e serviços que irá consumir. Contudo, a definição de qualidade de um serviço tornase difícil devido as suas características de intangibilidade, heterogeneidade e inseparabilidade (PARASURAMAN; ZEITHAML; BERRY, 2006).

Um serviço com qualidade, no entender de Las Casas (2006), é aquele capaz de proporcionar satisfação ao cliente. No entendimento de Corrêa e Caon (2006), oferecer uma proposta de valor real para o cliente faz parte da estratégia da empresa e é um item do sistema de serviços. A qualidade é pré-requisito para a consolidação da proposta. Manter a qualidade do serviço requer políticas, estratégias e a filosofia de melhoria contínua. Sempre se pode fazer melhor e incrementar a qualidade do serviço.

Segundo Lovelock e Wright (2001, p. 113), os clientes experimentam vários níveis de satisfação ou descontentamento após cada experiência de serviço de acordo com a medida na qual suas expectativas foram atendidas ou ultrapassadas. Considerando que a satisfação é um estado emocional, suas reações pós-compra podem envolver raiva, insatisfação, irritação, indiferença ou alegria.

Sabe-se também que clientes insatisfeitos podem criar problemas para a organização, pois além da tendência a migrar para a concorrência, acabam efetuando propaganda negativa através do boca a boca (LOVELOCK; WRIGHT, 2001). O relacionamento entre o cliente e a empresa é influenciado pelo resultado do processo de prestação de serviço. O que mantém a lealdade e sustenta o poder de competitividade no longo prazo é o atingimento e superação consistente das expectativas do cliente. Consumidor satisfeito implica em: redução do risco percebido, favorecendo a repetição da compra do serviço do mesmo fornecedor e possibilidade de recomendação do serviço a outros consumidores (CORRÊA; CAON, 2006).

Portanto, para que uma empresa obtenha qualidade na prestação de seus serviços, é necessário que seja construído um ambiente favorável na empresa, onde a excelência do serviço oferecido ao cliente, sendo um compromisso assumido por todos os membros integrantes da organização. Corroboram Lovelock e Wright (2001), afirmando que uma forma de manter esta excelência no serviço é estabelecer um processo constante de pesquisa, capaz de fornecer aos gerentes, informações úteis e oportunas.

Oferecer serviços de qualidade também é uma preocupação presente nas organizações hospitalares, de modo que, o hospital que recebe e trata bem seus pacientes, aumenta suas chances de ser bem sucedido, tanto no tratamento médico, quanto na formação de atitudes favoráveis em relação à sua imagem (MORETTO NETO; SILVA; SCHMITT, 2007). 
Segundo Klück et al. (2002), um paciente que internou em um hospital para realizar o diagnóstico e tratamento de uma doença complexa, mesmo saindo do hospital curado pode fazer uma má avaliação de seu tratamento, apenas pelo fato de que considerou as instalações ou o horário de visitas inadequados.

Gurgel Junior e Vieira (2002) observam que nas últimas décadas, houve uma mobilização em vários países em torno da aplicação de programas de qualidade nas organizações hospitalares, tendo como objetivo, incrementar seu gerenciamento e melhorar a eficiência de seus serviços. No entanto, Klück et al. (2002) mencionam que, por razões históricas, os hospitais brasileiros não vem respondendo adequadamente às necessidades de saúde da população.

\section{GESTÃO HOSPITALAR}

Moretto Neto, Silva e Schmitt (2007, p. 39), referem-se ao hospital, conforme a definição da organização Mundial da Saúde (OMS), como "parte integrante de uma organização médica e social cuja missão consiste em proporcionar assistência médica e de saúde completa, tanto curadora como preventiva e cujos serviços chegam ao âmbito familiar". Os autores mencionam ainda que o planejamento e a gestão da saúde, bem como do hospital, tem por objetivo aumentar ao máximo possível o estado de bem-estar de seus pacientes.

Essas instituições, no entendimento de Costa (1998, p. 153), evoluíram muito nas últimas décadas, podendo ser consideradas atualmente como "empresas modernas, que visam diminuir o sofrimento do paciente, permitindo que o mesmo permaneça o mínimo de tempo possível no ambiente hospitalar, afastado de seu lar e do convívio social".

A gestão hospitalar possui importância significativa, pois estas organizações estão entre as mais complexas, não só pela nobreza e amplitude de seu trabalho, mas principalmente por dispor de uma equipe de profissionais multidisciplinar com elevado grau de autonomia, para prestar assistência à saúde em caráter preventivo, curativo e reabilitador, com o auxilio de tecnologias de ponta no atendimento a pacientes em regime de internação, além de constituir um ambiente de prática de ensino-aprendizagem e produção científica (GURGEL JR.; VIEIRA, 2002).

\section{MÉTODOS SERVQUAL E SERVPERF}

Buscando compreender como os usuários percebiam e avaliavam a qualidade dos serviços, Parasuraman, Zeithaml e Berry (2006) investigaram doze grupos focais, onde os resultados obtidos demonstraram que os usuários são influenciados pelas dimensões do processo, e não só pelos resultados do serviço (CRUZ; MELLEIRO, 2010). Segundo os autores, o estudo revelou dez critérios avaliativos gerais, que são utilizados pelos usuários, independentemente do tipo de serviço prestado.

Porém, Mello et al. (2002) citam que ao desenvolver uma pesquisa posterior, estas dez dimensões - determinantes da qualidade em serviços - foram sintetizadas em apenas cinco, com o objetivo de melhorar suas propriedades psicométricas, tornando-as mais confiáveis e válidas. Estas cinco dimensões da qualidade são descritas por Freitas e Cozendey (2008, p. 5), adaptadas à avaliação de serviços hospitalares, conforme Quadro 01:

\begin{tabular}{|l|l|}
\hline \multicolumn{1}{|c|}{ Dimensão } & \multicolumn{1}{c|}{ Descrição da dimensão } \\
\hline Tangibilidade & $\begin{array}{l}\text { Refere-se à aparência de qualquer evidência física do serviço hospitalar, ou seja, a } \\
\text { aparência limpa ou a forma de se vestir dos funcionários, a limpeza das instalações, } \\
\text { atualização e inovação de novos equipamentos e facilidade no acesso às instalações. }\end{array}$ \\
\hline Confiabilidade & Confiabilidade de um serviço é a capacidade de prestar o serviço de forma confiável, \\
\hline
\end{tabular}




\begin{tabular}{|l|l|}
\hline & $\begin{array}{l}\text { precisa e consistente. No serviço hospitalar, a confiabilidade se traduz no conhecimento } \\
\text { técnico, na habilidade de executar um serviço que será prestado e na capacidade para } \\
\text { realizar o serviço prometido de forma segura e correta. }\end{array}$ \\
\hline $\begin{array}{l}\text { Atendimento e } \\
\text { Prontidão na } \\
\text { Resposta }\end{array}$ & $\begin{array}{l}\text { É a disposição de prestar os serviços hospitalares prontamente e auxiliar os clientes, } \\
\text { caracterizando-se por: agilidade no atendimento, eficiência em resolver os problemas, } \\
\text { atenção personalizada e a cortesia dos funcionários. }\end{array}$ \\
\hline $\begin{array}{l}\text { Garantia ou } \\
\text { Segurança }\end{array}$ & $\begin{array}{l}\text { Refere-se à isenção de qualquer perigo, risco ou problema. É uma dimensão } \\
\text { particularmente importante da qualidade do serviço hospitalar, pois, bem-estar e } \\
\text { segurança são considerações importantes, assim como o conhecimento e cortesia dos } \\
\text { funcionários e sua capacidade de inspirar confiança. }\end{array}$ \\
\hline Empatia & $\begin{array}{l}\text { A empatia fornece atenção individualizada a clientes que utilizam os serviços } \\
\text { hospitalares, buscando atender às suas necessidades específicas. Há necessidade } \\
\text { frequente de o cliente estar presente para que a prestação de serviço ocorra, haja } \\
\text { localização conveniente, acesso sinalizado, amplo horário de operações. }\end{array}$ \\
\hline
\end{tabular}

Quadro 01: Dimensões da qualidade

Fonte: Freitas e Cozendey (2008, p. 5)

O emprego do método SERVQUAL, conforme Freitas, Manhães e Cozendey (2006), é realizado em duas etapas: a primeira visa mensurar as expectativas dos clientes com relação ao serviço e, a segunda propõem-se a mensurar as percepções dos clientes acerca do desempenho do serviço. Os autores ressaltam ainda que as avaliações são realizadas através de um questionário, em geral utilizando-se de uma escala Likert de 7 pontos, na qual seus extremos variam entre "discordo totalmente" e "concordo totalmente".

Freitas, Manhães e Cozendey (2006) ainda citam que Cronin e Taylor (1992) investigaram a conceituação e mensuração da qualidade do serviço, bem como seu relacionamento com a satisfação dos consumidores e suas intenções de compra, sendo comparada a diferença de scores entre as expectativas e percepções, onde concluíram que a qualidade do serviço é mais bem avaliada quando utilizado apenas as percepções que os consumidores têm a respeito do desempenho do serviço. Com base nesta constatação, os autores mencionam que Cronin e Taylor (1992) propuseram um novo modelo de avaliação da qualidade, denominado SERVPERF, o qual se baseia no modelo SERVQUAL, porém, avalia apenas as medidas da percepção de desempenho do serviço.

Como aplicação empírica Freitas e Cozendey (2008) utilizaram o método SERVPERF com o objetivo de avaliar a qualidade dos serviços hospitalares prestados pelo Hospital São José do Avaí (HSJA), localizado na cidade de Itaperuma - RJ. Para a coleta de dados foi desenvolvido um formulário de pesquisa, composto por 24 itens, que utilizou uma escala Likert de 7 pontos, variando de "Discordo Fortemente" a " Concordo Fortemente". A coleta de dados ocorreu no período de novembro de 2006 a janeiro de 2007, sendo distribuídos 130 formulários aos pacientes das alas particular e convênios, onde retornaram 84 válidos.

A consistência interna do questionário foi avaliada através do coeficiente alfa de Cronbach. Para a análise dos resultados, os autores calcularam a média das percepções dos pacientes quanto ao desempenho do hospital à luz de cada item e para cada Dimensão. Por fim, utilizou-se a Análise dos Quartis para identificação dos itens mais críticos nos serviços prestados pelo hospital avaliado.

Após as análises, os autores concluíram que os pacientes, em geral, estão muito satisfeitos com os serviços prestados pelo HSJA, caracterizando um bom desempenho do hospital à luz dos itens considerados. Os autores observaram ainda que as dimensões "Atendimento" e "Confiabilidade" são as mais críticas desta instituição, ou seja, foram as que obtiveram os valores mais baixos em relação à qualidade dos serviços do HSJA. 


\section{CARACTERIZAÇÃO DA ORGANIZAÇÃO DO ESTUDO}

O Hospital Regional do Sudoeste - Walter Alberto Pécoits (HRS-WAP), está localizado no município de Francisco Beltrão - PR, e caracteriza-se por ser uma instituição pública de saúde, totalmente voltada ao atendimento dos usuários do Sistema Único de Saúde (SUS). Portanto, o HRS-WAP integra a rede de hospitais públicos, constituída pelo Governo do Estado do Paraná e mantida com recursos provenientes do orçamento da Secretaria de Estado da Saúde (SESA).

Sua construção teve início em janeiro de 2006, tendo sua implantação a partir da publicação da Lei Estadual 15.795/2008, intensificando-se em setembro de 2009 com a entrada da primeira equipe de colaboradores, contratados por meio do processo seletivo. $\mathrm{O}$ atendimento ao público iniciou em fevereiro de 2010.

Tendo um total de $12 \mathrm{mil} \mathrm{m}^{2}$ de área construída, disposta em um terreno de $50 \mathrm{mil} \mathrm{m}^{2}$, o HRS-WAP conta com 146 leitos de internamento, que são distribuídos da seguinte forma: a) clínica médica; b) clínica cirúrgica; c) obstetrícia; d) pediatria, e; e) Unidade de Terapia Intensiva (UTI). Também conta com uma unidade para atendimento de Urgência e Emergência, um Centro Cirúrgico com quatro salas para cirurgia geral e duas para cirurgia obstétrica, Ambulatório para consultas pré e pós-cirúrgicas, além de um Laboratório de Análises Clínicas e uma Central de Imagens. Esta estrutura é destinada a atender, prioritariamente, as necessidades dos 42 municípios pertencentes às microrregionais de saúde de Francisco Beltrão e Pato Branco. Também atende via Central Estadual de Leitos, pacientes oriundos de outras regiões do Estado. A organização dispõe de um corpo clínico composto por 121 profissionais médicos, além de 236 profissionais de enfermagem, de um total de 762 colaboradores.

Destaca-se ainda que o hospital não possui padrões de qualidade internos, definidos pela própria instituição ou pela SESA. No entanto, conforme a organização, o HRS-WAP deve atender aos padrões estabelecidos por órgãos de controle externos, como a Agencia Nacional de Vigilância Sanitária (ANVISA) e órgãos de classe: Conselho Regional de Medicina (CRM), Conselho Regional de Enfermagem (COREN), dentre outros.

\section{MÉTODO}

Esta pesquisa trata-se de um estudo exploratório que se utilizou tanto da abordagem qualitativa, quanto quantitativa. Em um primeiro momento, foi conduzida uma análise quantitativa, por meio da aplicação de um questionário, tendo como objetivo mensurar as percepções dos pesquisados sobre o atendimento. No segundo momento se fez uso da análise qualitativa, na qual os dados foram analisados de forma interpretativa, no intuito de proporcionar uma melhor compreensão do problema.

Ressalta-se que as considerações feitas acerca das informações coletadas tiveram o caráter particular, onde os resultados foram condizentes apenas com o universo da pesquisa, não sendo possível generalizá-las às demais organizações de mesma atividade.

A pesquisa qualitativa, na concepção de Malhotra (2006), trata-se de um método de pesquisa não estruturada, de caráter exploratório e que proporciona uma melhor percepção e compreensão do contexto do problema. McDaniel; Gates (2006) contribuem citando que o uso deste tipo de pesquisa é a melhor forma para compreender, a fundo, motivações e sentimentos dos consumidores. Já a pesquisa quantitativa procura traduzir as informações coletadas em números, para posteriormente classificá-las e analisá-las com o auxilio de recursos e técnicas estatísticas (FÁVERO et al., 2009). 
Como procedimento para coleta de dados, foi utilizado o método de levantamento, já que a proposta da pesquisa é verificar as opiniões (percepções) dos pesquisados. $\mathrm{O}$ levantamento, no entendimento de Malhotra (2006, p. 182), "envolve um questionário estruturado que os entrevistados devem responder e que foi feito para elucidar informações específicas". O autor ainda cita que este método é baseado no interrogatório dos participantes, sendo feitas várias perguntas com o intuito de verificar seu perfil, comportamento, percepções e motivações, dentre outras informações relevantes.

O estudo foi realizado no HRS-WAP, sendo utilizada uma amostra não-probabilística e intencional, levando-se em consideração alguns critérios como: aceitação espontânea dos pacientes; serem maiores de 18 anos; que apresenta-se em condições clínicas favoráveis, e; estivessem internados nas unidades de clínicas médica ou cirúrgica.

\section{COLETA DE DADOS}

Para realizar a coleta de dados, utilizou-se um questionário baseado no modelo SERVPERF, composto por duas partes: a primeira visou identificar os dados inerentes ao perfil do entrevistado e, a segunda teve como objetivo levantar as percepções dos mesmos sobre 25 elementos que compõem as cinco dimensões da qualidade propostas por Parasuramam et al. (2006): Tangibilidade, Confiabilidade, Responsabilidade, Garantia e Empatia. Ao final, foi adicionada uma questão $\left(\mathrm{I}_{26}\right)$ indagando os pacientes sobre seu nível geral de satisfação com os serviços do HRS-WAP. Para facilitar a ponderação das respostas, utilizou-se de uma escala Likert de 7 pontos.

Com o intuito de aprimorar o instrumento de pesquisa, proporcionando maior qualidade aos dados coletados, sugeriu-se a realização do pré-teste do instrumento, entre os dias 08 e 09 de outubro de 2011, onde foram abordados 6 pacientes, compondo a amostragem utilizada para avaliação do instrumento. A partir deste pré-teste foi observada a necessidade de adequação de alguns elementos do instrumento de coleta. Depois de feitas as adequações no instrumento, realizou o levantamento entre os dias 10 a 21 de outubro.

A abordagem foi realizada sempre nos leitos, com o pesquisador portando crachá de identificação. A fim de esclarecer os pacientes a cerca dos objetivos da pesquisa e minimizar possíveis constrangimentos, antes da aplicação de cada questionário, foi entregue o Termo de Consentimento Livre e Esclarecido (TCLE), conforme exigência do Comitê de Ética em Pesquisa da Secretaria de Estado da Saúde (SESA). O tempo médio de aplicação do questionário foi de aproximadamente 20 minutos por paciente. Foram abordados 56 pacientes das clínicas médica e cirúrgica. O pesquisador compareceu sempre ao final da tarde, tendo o cuidado para não aplicar o questionário no horário de visita ou de alimentação.

\section{APRESENTAÇÃO, ANÁLISE E DISCUSSÃO DOS RESULTADOS}

Para a análise, inicialmente os dados foram organizados e tabulados com o auxilio da estatística descritiva, para que pudessem ser mensuradas as médias perceptivas dos pesquisados.

\section{CONFIABILIDADE DO INSTRUMENTO}

Para verificar a confiabilidade do instrumento de pesquisa, foi utilizado o coeficiente alpha $(\alpha)$ de Cronbach. Conforme Freitas e Cozendey (2008), o $\alpha$ de Cronbach é uma das mais tradicionais estimativas para verificação da confiabilidade de um questionário que tenha 
sido aplicado em uma pesquisa. Os autores ainda explicam que valores de alpha menores ou iguais a 0,6 , geralmente, representam confiabilidade de consistência interna insatisfatória. Os valores de $\alpha$ para cada item do instrumento podem ser observados na Tabela 01 .

Tabela 01 - Análise da confiabilidade do instrumento

\begin{tabular}{|c|c|c|c|c|}
\hline Dimensão & Item & $\boldsymbol{P}$ & $P($ Dimensão $)$ & Alpha de Cronbach \\
\hline \multirow{5}{*}{ Tangibilidade } & $\mathrm{I}_{1}$ & 6,80 & \multirow{5}{*}{6,93} & 0,9278 \\
\hline & $\mathrm{I}_{2}$ & 6,95 & & 0,9268 \\
\hline & $\mathrm{I}_{3}$ & 6,98 & & 0,9251 \\
\hline & $\mathrm{I}_{4}$ & 6,96 & & 0,9252 \\
\hline & $\mathrm{I}_{5}$ & 6,93 & & 0,9239 \\
\hline \multirow{5}{*}{ Confiabilidade } & $\mathrm{I}_{6}$ & 6,95 & \multirow{5}{*}{6,89} & 0,9232 \\
\hline & $\mathrm{I}_{7}$ & 6,91 & & 0,9222 \\
\hline & $\mathrm{I}_{8}$ & 6,95 & & 0,9205 \\
\hline & $\mathrm{I}_{9}$ & 6,79 & & 0,9198 \\
\hline & $\mathrm{I}_{10}$ & 6,88 & & 0,9252 \\
\hline \multirow{5}{*}{ Responsabilidade } & $\mathrm{I}_{11}$ & 6,82 & \multirow{5}{*}{6,86} & 0,9186 \\
\hline & $\mathrm{I}_{12}$ & 6,89 & & 0,9203 \\
\hline & $\mathrm{I}_{13}$ & 6,95 & & 0,9226 \\
\hline & $\mathrm{I}_{14}$ & 6,86 & & 0,9223 \\
\hline & $\mathrm{I}_{15}$ & 6,79 & & 0,9227 \\
\hline \multirow{5}{*}{ Garantia } & $\mathrm{I}_{16}$ & 6,91 & \multirow{5}{*}{6,86} & 0,9210 \\
\hline & $\mathrm{I}_{17}$ & 6,84 & & 0,9204 \\
\hline & $\mathrm{I}_{18}$ & 6,89 & & 0,9191 \\
\hline & $\mathrm{I}_{19}$ & 6,84 & & 0,9200 \\
\hline & $\mathrm{I}_{20}$ & 6,84 & & 0,9233 \\
\hline \multirow{5}{*}{ Empatia } & $\mathrm{I}_{21}$ & 6,82 & \multirow{5}{*}{6,91} & 0,9240 \\
\hline & $\mathrm{I}_{22}$ & 6,89 & & 0,9226 \\
\hline & $\mathrm{I}_{23}$ & 6,89 & & 0,9198 \\
\hline & $\mathrm{I}_{24}$ & 6,96 & & 0,9232 \\
\hline & $\mathrm{I}_{25}$ & 6,96 & & 0,9238 \\
\hline- & $\mathrm{I}_{26}$ & 6,88 & - & 0,9249 \\
\hline
\end{tabular}

Fonte: Dados da pesquisa (2011).

O cálculo do $\alpha$ (alpha) foi realizado com o auxilio do programa estatístico Statistical Package for the Social Sciences - SPSS, versão 15. Com base nos dados avaliados, verificouse um valor de alpha de 0,9254 para o instrumento, além de valores de alpha superiores a 0,91 em todos os itens do questionário, representando um nível de confiabilidade elevado. 


\section{CARACTERIZAÇÃO DA AMOSTRA}

A primeira seção do questionário foi destinada para a caracterização do perfil da amostra estudada, sendo avaliados os seguintes elementos: sexo, idade, escolaridade, município de residência, localização da residência e unidade de atendimento.

Quanto ao gênero dos pesquisados pode ser observada uma distribuição razoavelmente equilibrada entre homens (54\%) e mulheres (46\%), com participação pouco superior do grupo masculino, que condiz com a média apresentada pela instituição para os dois estratos avaliados. Quanto à idade dos respondentes, 36\% possuem acima de 65 anos; $21 \%$ entre 65 e 54 anos; $20 \%$ entre 29 e 18 anos; $14 \%$ entre 41 e 30 anos, e 9\% entre 53 e 42 anos. Nota-se uma presença acentuada de pacientes com idade mais avançada, sendo que $57 \%$ dos pacientes abordados possuem mais de 54 anos. Segundo informações da instituição, isso pode ser justificado pelo fato de pessoas nesta faixa etária estarem mais vulneráveis a doenças e acidentes domésticos.

Ao se questionar o grau de escolaridade de cada participante na pesquisa ficou evidenciado um baixo nível de escolaridade na amostra avaliada, onde $75 \%$ dos entrevistados, sendo representados por pessoas que apenas frequentaram os anos iniciais de estudo ou os que não foram alfabetizados. Conforme a instituição, isso reflete o perfil dos usuários do Sistema Único de Saúde (SUS).

Quanto aos municípios onde residem os pacientes entrevistados, percebeu-se a grande área de abrangência do HRS, demonstrando a sua importância para a região, conforme demonstrado na Figura 01.

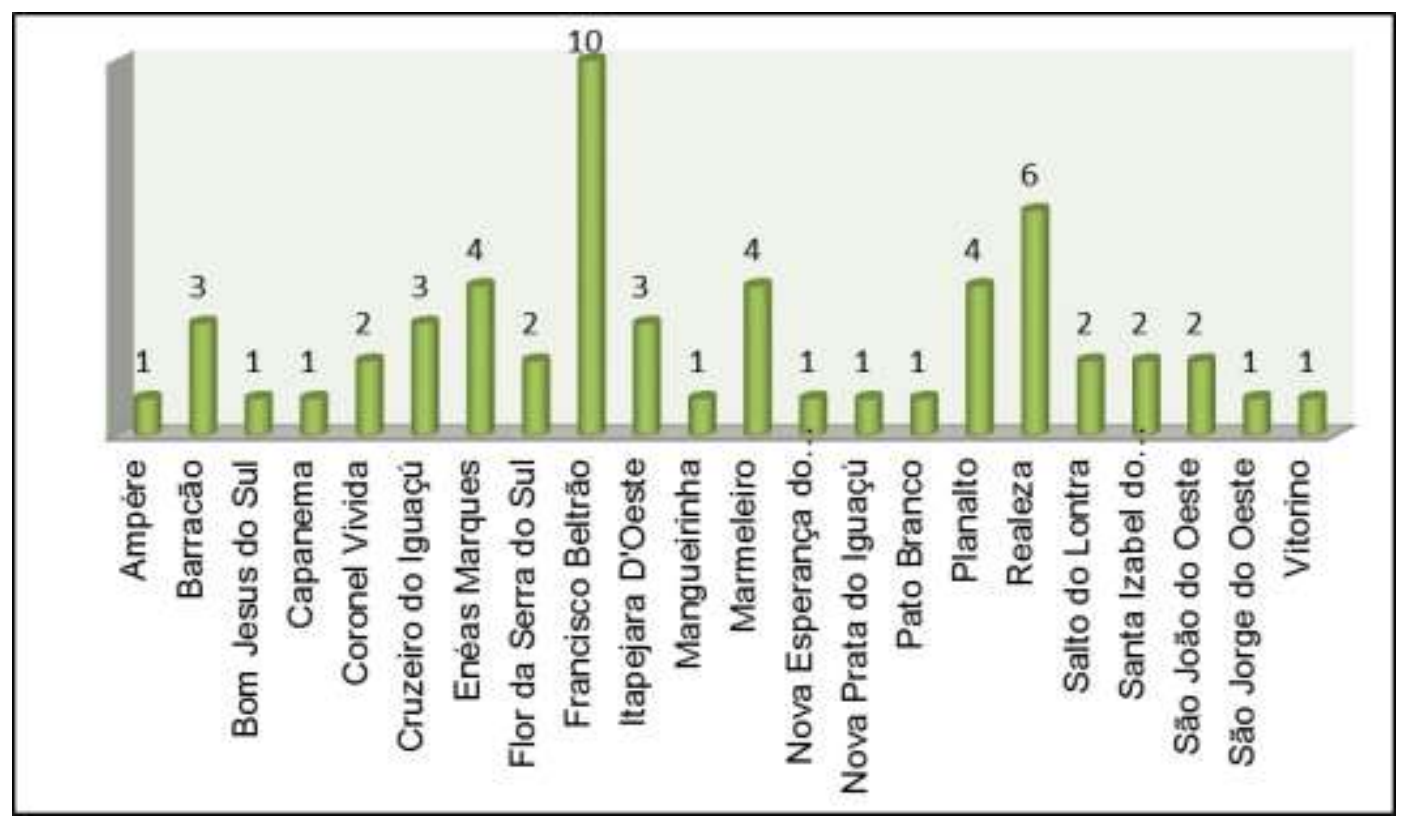

Figura 01 - Município de residência do grupo avaliado

Fonte: Dados da pesquisa (2011).

Nota-se uma distribuição bastante diversificada, com pacientes oriundos de vários municípios da região, o que confirma a atuação regional desta instituição de saúde no atendimento à demanda por procedimentos de média e alta complexidade de todos os municípios do Sudoeste do Paraná, e de outras regiões por meio da Central de Leitos. Também foi constatado que $70 \%$ dos entrevistados residem na área urbana, enquanto que $30 \%$ são provenientes da área rural. 
Quanto à representatividade de pesquisados por unidade de atendimento do HRS, a pesquisa configurou que a clínica cirúrgica respondeu por $2 / 3$ dos pacientes entrevistados. De acordo com a instituição, isso ocorre por dois motivos: $1^{\circ}$ ) os pacientes da clínica médica geralmente permanecem internados por um período maior; $2^{\circ}$ ) a clínica cirúrgica dispõe de 49 leitos, enquanto que a clínica médica dispõe de apenas 21.

Pode-se observar que a amostra estudada consiste em um público bastante heterogêneo, mas que tem em comum um baixo nível de escolaridade. Essa característica atua diretamente no senso perceptível das pessoas, pois está ligado a seus valores e ao conhecimento implícito formado individualmente.

\section{APRESENTAÇÃO E ANÁLISE DOS DADOS}

Após a análise dos dados observou-se que todas as variáveis avaliadas obtiveram um bom desempenho, com médias elevadas, sendo as com melhor desempenho respectivamente: Tangibilidade (6,93), Empatia (6,91), Confiabilidade (6,89), Garantia (6,86) e Responsabilidade $(6,86)$.

O bom desempenho da variável Tangibilidade deve-se, principalmente, pelo fato do hospital dispor de uma estrutura nova, com pouco menos de dois anos de uso, e que, além de ser a unidade estadual mais recente, é tratada como modelo estadual em termos de estrutura. Isto ficou evidenciado a partir de conversas paralelas com os pacientes, durante a aplicação do questionário, onde os mesmos relataram estar muito satisfeitos com a infraestrutura do hospital, já que as unidades onde eram atendidos anteriormente, principalmente em seus municípios de origem, possuem estruturas desgastadas, mal higienizadas e incapazes de oferecer um mínimo de conforto a seus usuários.

No caso da variável Empatia, seu desempenho pode ser atribuído a um corpo de trabalho novo, admitido a pouco mais de seis meses, e que ainda encontra-se plenamente motivado com o trabalho. Conforme apresentado pelos pacientes, os funcionários do HRSWAP, principalmente médicos e pessoal de enfermagem, são muito gentis e atenciosos, além de atenderem prontamente quando solicitados. Isso contrasta com o apresentado pelos mesmos para outras unidades públicas onde, em geral, há demora no atendimento, bem como desatenção e falta de interesse por parte de funcionários em ajudar os pacientes.

A Figura 02 apresenta o nível de satisfação obtido para cada elemento do questionário. 


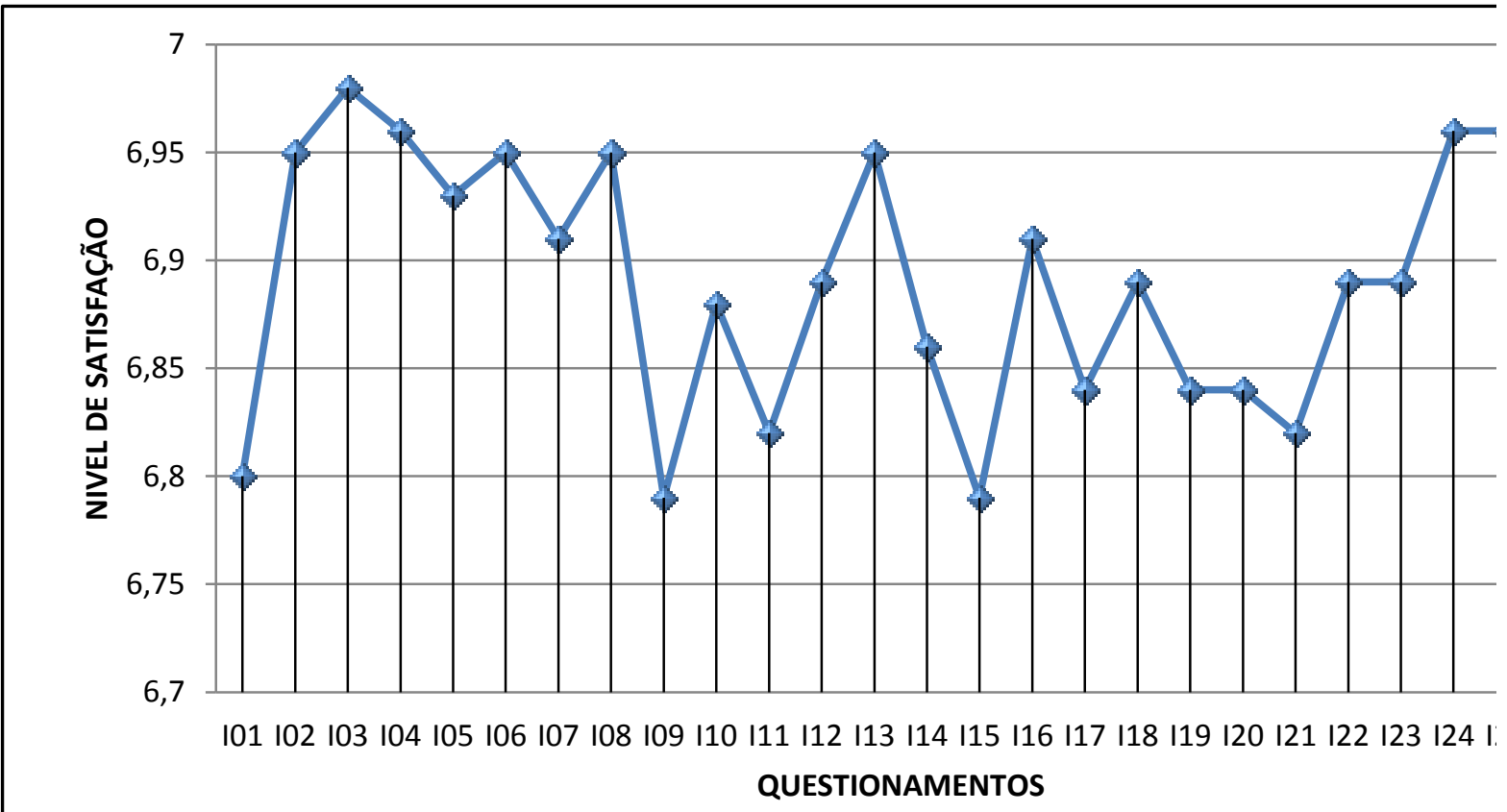

Figura 02 - Nível de satisfação por cada elemento questionado referente ao serviço.

Fonte: Dados da pesquisa (2011).

$\mathrm{Na}$ variável tangibilidade, que foi a que mais se destacou, obtendo quatro notas superiores a 6,90 dentre os cinco elementos que a compõe, a variável que mais se destacou foi o acesso $\left(\mathrm{I}_{03}\right)$, com nota 6,98 , quase atingindo o limite máximo do instrumento. Na sequência estão iluminação $\left(\mathrm{I}_{04}\right)$, limpeza $\left(\mathrm{I}_{02}\right)$ e conforto $\left(\mathrm{I}_{05}\right)$, com 6,96, 6,95 e 6,93 pontos, respectivamente. Já o elemento com menor desempenho nesta variável foi o visual das instalações $\left(\mathrm{I}_{01}\right)$, com nota 6,80 .

No que diz respeito à confiabilidade, os elementos que mais se destacaram foram o pontualidade $\left(\mathrm{I}_{06}\right)$ e a realização correta do serviço $\left(\mathrm{I}_{08}\right)$, ambos atingindo média 6,95 . A seguir temos interesse em resolver os problemas $\left(\mathrm{I}_{07}\right)$ com 6,91 e realização do serviço conforme o prometido $\left(\mathrm{I}_{10}\right)$ com 6,88 , enquanto que o elemento com menor desempenho foi o fornecimento correto de informações $\left(\mathrm{I}_{09}\right)$, com 6,79 pontos.

Para a variável responsabilidade, que foi a que obteve a média mais inferior, juntamente com garantia, cabe destaque para o elemento disposição dos funcionários $\left(\mathrm{I}_{13}\right)$, cuja média atingiu 6,95. Na sequência estão atendimento imediato $\left(\mathrm{I}_{12}\right)$, informação sobre o estado de saúde e tratamento $\left(\mathrm{I}_{14}\right)$ e informação acerca dos serviços a serem realizados $\left(\mathrm{I}_{11}\right)$, respectivamente com 6,89, 6,86 e 6,82 pontos. Já o elemento com menor pontuação foi a capacidade dos funcionários em esclarecer dúvidas $\left(\mathrm{I}_{15}\right)$, com 6,79 de média, sendo, juntamente com o item $\mathrm{I}_{09}$, o menor valor obtido dentre os elementos do questionário.

Quanto à variável garantia, temos como destaque o item comportamento confiável dos funcionários $\left(\mathrm{I}_{16}\right)$, que somou 6,91 pontos, sendo seguido por segurança em utilizar os serviços do hospital $\left(\mathrm{I}_{18}\right)$ com 6,89. Quanto aos elementos bem-estar $\left(\mathrm{I}_{17}\right)$, conhecimento demonstrado $\left(\mathrm{I}_{19}\right)$ e resolução dos problemas $\left(\mathrm{I}_{20}\right)$, estes foram os que obtiveram as menores somatórias, ambos atingindo 6,84 pontos.

No que tange à empatia, destacaram-se os itens atenção $\left(I_{24}\right)$ e gentileza dos funcionários $\left(\mathrm{I}_{25}\right)$, com 6,96 pontos, sendo seguido por atendimento pessoal $\left(\mathrm{I}_{22}\right)$ e interesse $\left(\mathrm{I}_{23}\right)$, que somaram 6,89. Já o item entendimento das necessidades $\left(\mathrm{I}_{21}\right)$, obteve a menor somatória desta variável, com 6,82 pontos.

Por fim, a variável extra, que buscou avaliar de forma geral a satisfação dos pacientes com os serviços prestados pelo HRS-WAP, atingiu 6,88 pontos de somatória, muito próximo 
ao valor obtido a partir da média dos 25 elementos que compõem o instrumento, que foi de 6,89 .

Como podem ser observados, todos os elementos obtiveram um nível de satisfação elevado, próximos à máxima permitida pelo instrumento, cabendo destaque aos itens Acesso $\left(\mathrm{I}_{03}\right)$, Iluminação $\left(\mathrm{I}_{04}\right)$, Atenção dos funcionários $\left(\mathrm{I}_{24}\right)$ e Gentileza dos funcionários $\left(\mathrm{I}_{25}\right)$. Isso demonstra que, atualmente, o HRS-WAP está sendo eficaz no que diz respeito a satisfazer as necessidades de seus pacientes.

A Figura 03 faz uma relação entre o nível de satisfação do grupo feminino e do grupo masculino, obtido para cada elemento do questionário.

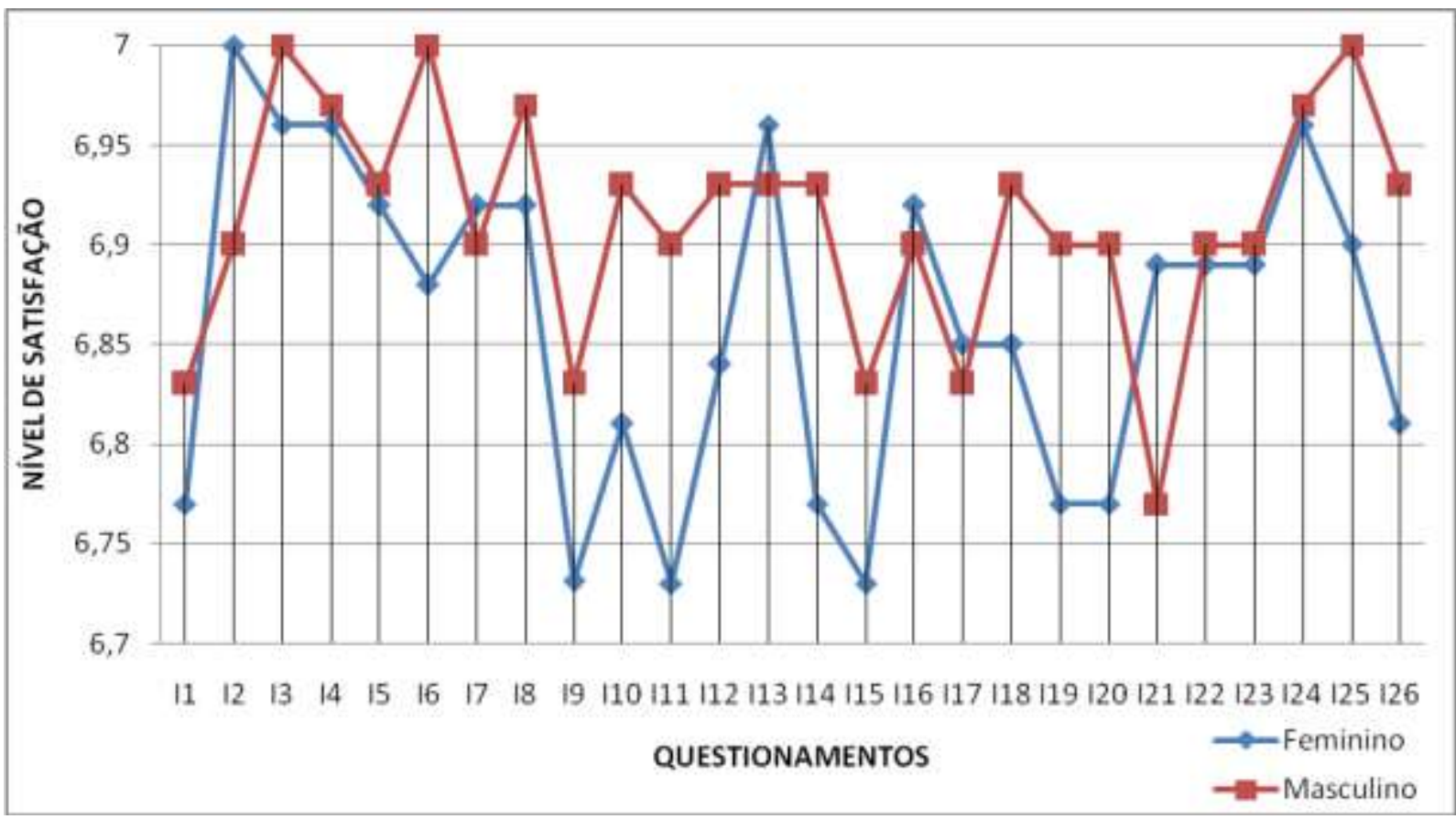

Figura 03 - Nível de satisfação feminino/masculino à luz de cada elemento.

Fonte: Dados da pesquisa (2011).

Como pode ser observado, o grupo masculino apresentou valores superiores ao grupo feminino na maioria dos elementos do questionário, obtendo assim uma média ligeiramente superior, sendo 6,91 pontos para eles contra 6,86 pontos para elas.

O nível de satisfação do grupo masculino só foi inferior nos itens: limpeza $\left(\mathrm{I}_{02}\right)-(-$ $0,10)$; interesse em resolver os problemas $\left(\mathrm{I}_{07}\right)-(-0,02)$; disposição dos funcionários $\left(\mathrm{I}_{13}\right)-(-$ $0,03)$; comportamento confiável dos funcionários $\left(\mathrm{I}_{16}\right)-(-0,02)$; bem-estar $\left(\mathrm{I}_{17}\right)-(-0,01)$, e; entendimento das necessidades $\left(\mathrm{I}_{21}\right)-(-0,12)$. Em todos os demais itens, o grupo masculino obteve um valor superior ao feminino, cabendo destaque para: informação acerca dos serviços a serem realizados $\left(I_{11}\right)-(+0,17)$, e; informação sobre o estado de saúde e tratamento $\left(I_{14}\right)$ $(+0,16)$.

A Figura 04 faz uma relação entre o nível de satisfação dos pacientes atendidos na clínica médica e dos atendidos na clínica cirúrgica, obtido para cada elemento do questionário. 


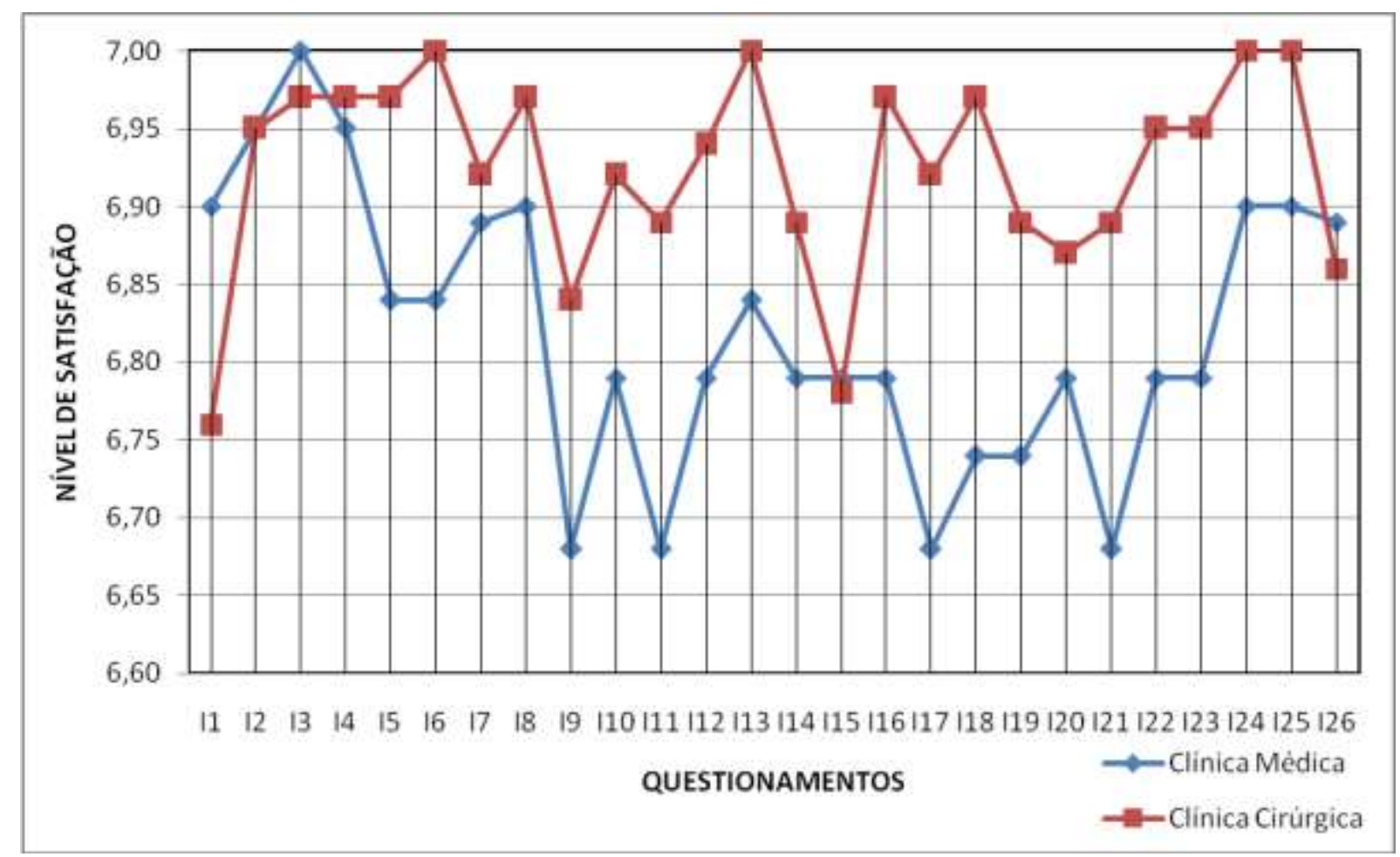

Figura 04 - Nível de satisfação clínica médica/clínica cirúrgica à luz de cada elemento.

Fonte: Dados da Pesquisa (2011).

Neste caso observa-se que, embora ainda pequena, há uma diferença pouco mais significativa que na relação anterior, ficando evidente que o nível de satisfação dos pacientes da clínica cirúrgica é superior ao dos pacientes da clínica médica, sendo as médias obtidas 6,93 e 6,82 respectivamente. Destaca-se também o fato de a clínica cirúrgica obter nota 7,00, que é a máxima do instrumento, em quatro elementos: pontualidade ( $\left.\mathrm{I}_{06}\right)$; disposição dos funcionários $\left(\mathrm{I}_{13}\right)$; atenção $\left(\mathrm{I}_{24}\right)$, e; gentileza dos funcionários $\left(\mathrm{I}_{25}\right)$.

O nível de satisfação dos pacientes da clínica cirúrgica só foi inferior nos itens: visual das instalações $\left(\mathrm{I}_{01}\right)-(-0,14)$; acesso $\left(\mathrm{I}_{03}\right)-(-0,03)$, e; capacidade dos funcionários em esclarecer dúvidas $\left(\mathrm{I}_{15}\right)$ - (-0,01). Em todos os demais itens seu desempenho foi superior, destacando-se: informação acerca dos serviços a serem realizados $\left(\mathrm{I}_{11}\right)-(+0,21)$; bem-estar $\left(\mathrm{I}_{17}\right)$ - (+0,23); segurança em utilizar os serviços do hospital $\left(\mathrm{I}_{18}\right)-(+0,24)$, e; entendimento das necessidades $\left(\mathrm{I}_{21}\right)-(+0,21)$.

Contudo, essa diferença pode ser resultado do tempo de internação, já que a clínica cirúrgica trabalha com pacientes que realizam cirurgias e permanecem no hospital por um curto período de tempo, em média três dias, segundo informações da instituição. Já a clínica médica recebe pacientes que necessitam realizar tratamentos mais prolongados, podendo permanecer internados por semanas, ou até mesmo meses. Esse período de internamento mais prolongado infere diretamente no senso crítico dos pacientes, empurrando o nível de satisfação para baixo.

A Figura 05 ilustra uma relação entre o nível de satisfação dos pacientes que possuem um nível de instrução baixo, ou que não chegaram a concluir o ensino médio, e dos pacientes que concluíram o ensino médio e/ou continuaram seus estudos, obtido para cada elemento do questionário. 


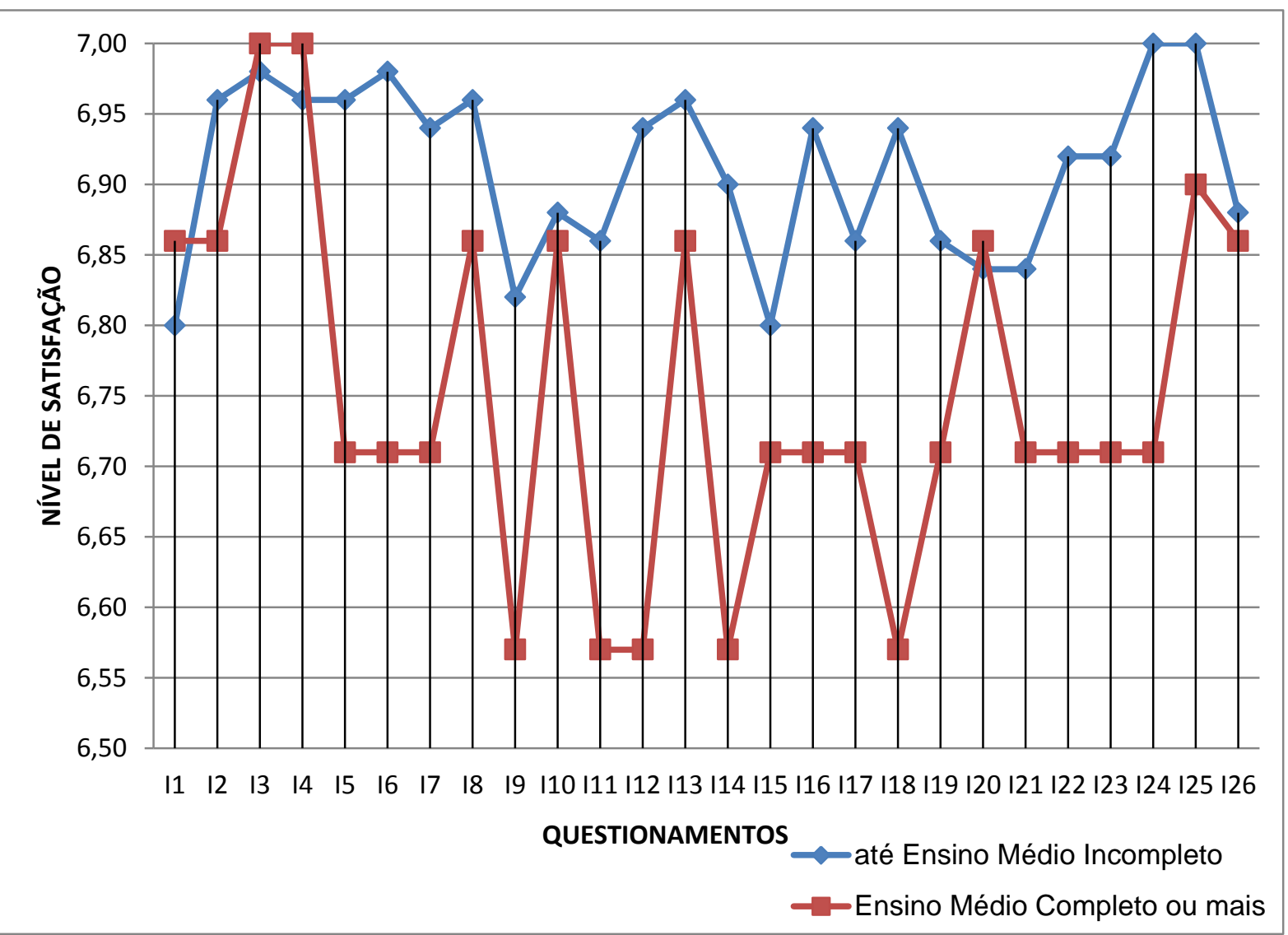

Figura 05 - Nível de satisfação com relação ao nível de instrução à luz de cada elemento.

Fonte: Dados da Pesquisa (2011).

Nesta relação observa-se uma variação mais significativa que nas duas anteriores, sendo que os pacientes que informaram ter formação até o ensino médio incompleto apresentaram média de 6,91 pontos, enquanto que os pacientes com ensino médio completo ou mais obtiveram uma média de 6,75. Importante destacar que o grupo de pacientes que possuem formação até o ensino médio incompleto apresentou, em todos os elementos, ponderações iguais ou superiores a 6,80 , enquanto que o grupo com ensino médio completo ou mais somou 16 notas iguais ou inferiores a 6,71 .

O nível de satisfação dos pacientes com menor instrução só obteve ponderação menor em quatro elementos do questionário: instalações $\left(\mathrm{I}_{01}\right)-(-0,06)$; acesso $\left(\mathrm{I}_{03}\right)-(-0,02)$; iluminação $\left(\mathrm{I}_{04}\right)-(-0,04)$, e; resolução dos problemas $\left(\mathrm{I}_{20}\right)-(-0,02)$. Nos demais itens o desempenho foi superior, cabendo destaque para: atendimento imediato $\left(\mathrm{I}_{12}\right)-(+0,37)$; informação sobre o estado de saúde e tratamento $\left(\mathrm{I}_{14}\right)$ - $(+0,33)$, e; segurança em utilizar os serviços do hospital $\left(\mathrm{I}_{18}\right)-(+0,37)$. Estas médias podem ser justificadas pelo fato de pessoas com um nível de instrução maior, geralmente serem mais críticos quanto à qualidade do atendimento.

\section{ANÁLISE DOS PONTOS CRÍTICOS}

Embora os resultados obtidos tenham sido muito positivos, é necessário identificar os elementos cujo desempenho tenha ficado a baixo da média, para que desta forma possam ser tomadas ações para a melhoria do serviço prestado pela organização pesquisada. A partir dos dados apresentados anteriormente, percebe-se que Garantia e Responsabilidade constituem 
as dimensões mais críticas, ou seja, são as dimensões que mais contribuem negativamente para a qualidade do serviço do HRS-WAP.

Além disso, uma análise mais detalhada é recomendada com o intuito de identificar a contribuição de cada item pertencente a cada dimensão, pois, eventualmente, uma dimensão pode não ter sido considerada mais crítica, porém esta mesma dimensão pode ter um ou mais elementos que apresentam elevado grau de discordância.

Neste sentido, utilizou-se a Análise dos Quartis para identificar os itens mais críticos do serviço, os quais devem ter ações corretivas e preventivas priorizadas. A Figura 06 apresenta a classificação da prioridade dos itens.

\begin{tabular}{|c|c|c|c|c|c|c|c|c|c|c|c|c|c|c|c|c|c|c|c|c|c|c|}
\hline \multicolumn{23}{|c|}{ Classificação da Prioridade dos Itens segundo as Percepções do Desempenho } \\
\hline \multicolumn{5}{|c|}{ Prioridade Crítica } & \multicolumn{6}{|c|}{ Prioridade Alta } & \multicolumn{5}{|c|}{ Prioridade Moderada } & \multicolumn{7}{|c|}{ Prioridade Baixa } \\
\hline L & $I_{15}$ & I $I_{41}$ & I. 12 & $\mathrm{I}_{17}$ & $\mathbf{I}_{19}$ & $I_{30}$ & $I_{14}$ & \begin{tabular}{l|l}
$I_{10}$ & 1
\end{tabular} & \begin{tabular}{l|l}
$I_{12}$ & $I_{1}$
\end{tabular} & $\mathbf{I}_{18} \mathbf{I}$ & \begin{tabular}{l|l}
$I_{22}$ & $I_{2}$
\end{tabular} & $I_{23}$ & \begin{tabular}{l|l}
$I_{7}$ & $I_{16}$
\end{tabular} & $\mathbf{I}_{5}$ & $\mathrm{I}_{2}$ & $\mathrm{I}_{6}$ & $\mathrm{I}_{8}$ & $I_{13}$ & $\mathrm{I}_{4}$ & $\mathrm{I}_{24}$ & $I_{25}$ & $\mathrm{I}_{3}$ \\
\hline & 6,79 & $6,80 \cdot 6,8$ & 32.6 .82 & & 6,84 & 6,846 & 6,866 & 6,886 & $6,896,8$ & 6,896 & $6,896,8$ & \begin{tabular}{l|l|l}
6,89 & 6,9
\end{tabular} & \begin{tabular}{l|l}
91 & 6191
\end{tabular} & 6,93 & 6,95 & 6,95 & 6,956 & 6,956 & \begin{tabular}{l|l}
6,96 & 6,
\end{tabular} & \begin{tabular}{l|l}
6,96 & 6
\end{tabular} & $6,96 \mid 6$ & 6,98 \\
\hline & & & $1^{0} \mathrm{Qu}$ & uartil = & $=6,84$ & & & & $2^{\circ}$ Quar & artil $=6$ & 6,89 & & & $3^{0} \mathrm{Qu}$ & uartil = & $=6,95$ & & & & & & \\
\hline
\end{tabular}

Figura 06 - Regiões críticas e classificação de prioridades segundo as percepções do desempenho. Fonte: Dados da Pesquisa (2011).

Como pode ser observado, dentre os itens considerados mais críticos, e que necessitam ter ações corretivas priorizadas, três elementos dizem respeito às informações, ou seja, os pacientes estão mais insatisfeitos no que se refere ao fornecimento de informações. Portanto, sugere-se que os servidores deem mais atenção à transmissão de informações aos pacientes, de modo que estes possam ter um melhor esclarecimento acerca dos procedimentos que estão sendo realizados durante sua estada no hospital.

Também se observou que, embora a variável Tangibilidade tenha obtido o melhor desempenho na comparação entre as variáveis, na análise individual dos elementos o visual das instalações físicas encontra-se como uma questão com prioridade crítica, havendo a necessidade de desenvolver ações que melhorem a aparência física do hospital.

Outra questão crítica está relacionada ao entendimento das necessidades dos pacientes e o bem-estar dos mesmos. Orienta-se, neste caso, que os servidores dêem mais atenção pessoal aos pacientes, de modo a entender melhor suas dificuldades, buscando criar condições que minimizem o desconforto gerado durante o internamento.

\section{CONSIDERAÇÕES FINAIS}

Ao final do estudo, realizado no Hospital Regional do Sudoeste WAP (HRS-WAP), observou-se que esta instituição oferece serviços de alta qualidade, verificada a partir da obtenção de níveis elevados de satisfação apresentados pelos pacientes abordados na pesquisa. Salienta-se que os resultados apresentados dizem respeito apenas ao HRS-WAP, não podendo esta análise ser utilizada como um contexto geral, ou seja, como parâmetro para descrever os serviços de outras instituições de saúde, tanto públicas, quanto privadas.

De um modo geral, o HRS-WAP obteve um bom desempenho, sendo que todos os elementos avaliados obtiveram um nível de satisfação elevado, muito próximos da máxima do instrumento. Cabe destaque às variáveis Tangibilidade e Empatia, que apresentaram as melhores médias, consequência de uma estrutura física nova e capaz de proporcionar conforto aos usuários, além de dispor de um corpo de trabalho motivado e que atende com prontidão e respeito ao paciente. 
Quando se comparou as unidades de internamento, pode-se perceber que os pacientes atendidos na clínica cirúrgica estão mais satisfeitos do que os pacientes da clínica médica. Contudo, essa diferença pode ser resultante do tempo de internação, já que os pacientes da clínica médica permanecem, em geral, por períodos muito mais longos, influenciando na redução do nível de satisfação.

Sendo feita a relação entre o nível de satisfação e o nível de instrução, nota-se que quanto maior a formação do paciente, maior a tendência de retração no valor atribuído ao serviço. Isto por que, geralmente, quanto maior a formação do paciente, mais crítico ele será quanto à qualidade do serviço.

Ao final, realizou-se a análise dos Quartis, para verificar os pontos mais críticos do serviço, ou seja, os pontos que necessitam de ações corretivas prioritárias. Desta forma pode ser notado que a questão de maior relevância consiste na transmissão de informações ao paciente, a qual deve ter maior atenção por parte dos servidores.

Também, como o HRS-WAP foi fundado recentemente e está com sua capacidade sendo utilizada parcialmente, em cerca de $60 \%$, podendo ser este um dos principais motivos que influenciaram para a obtenção de notas elevadas, sugere-se que seja realizado um novo estudo, em um novo recorte temporal, para verificar a evolução dos indicadores e avaliar se o hospital conseguiu manter este nível elevado ou obteve regressão. Para ampliar as discussões, sugere-se a realização de um estudo comparativo com os outros Hospitais Regionais do Estado, para verificar se estes possuem índices similares.

\section{REFERÊNCIAS}

BRASIL. Constituição (1988). Constituição da República Federativa do Brasil: promulgada em 5 de outubro de 1988. 38 ed. São Paulo: Saraiva, 2006.

CORRÊA, H. L.; CAON, M. Gestão de serviços: lucratividade por meio de operações e de satisfação dos clientes. São Paulo: Atlas, 2006.

COSTA, H. Tópicos de Administração Hospitalar. São Paulo: Renovarum, 1998.

CRUZ, V. B. S.; MELLEIRO, M. M. Análise da satisfação dos usuários de um hospital privado. Revista da Escola de Enfermagem da USP, v. 44, n. 1, p. 147-153, 2010.

FÁVERO, L. P.; BELFIORE, P.; SILVA, F. L.; CHAN, B. L. Análise de Dados: modelagem multivariada para tomada de decisões. Rio de Janeiro: Campus Elsevier, 2009. 646 p.

FREITAS, A. L. P.; COZENDEY, M. I. Um modelo SERVPERF para avaliação de serviços hospitalares. In: ENCONTRO NACIONAL DE ENGENHARIA DA PRODUÇÃO - Enegep, XXVIII, 2008, Rio de Janeiro. Anais... Disponível em: <http://www.abepro.org.br/biblioteca/enegep2008_TN_STO_070_502_10664.pdf>. Acesso em: Ago. 2011.

FREITAS, A. L. P.; MANHÃES, N. R. C.; COZENDEY, M. I. Emprego do SERVQUAL na avaliação da Qualidade de Serviços de Tecnologia da Informação: uma análise experimental. In: ENCONTRO NACIONAL DE ENGENHARIA DA PRODUÇÃO - Enegep, XXVI, 2006, Fortaleza. Anais... Disponível em: <http://www.abepro.org.br/biblioteca/ ENEGEP2006_TR530352_7613.pdf>. Acesso em: Jun. 2011. 
GURGEL JUNIOR, G. D.; VIEIRA, M. M. F. Qualidade total e administração hospitalar: explorando disjunções conceituais. Ciência \& Saúde Coletiva, 7(2), pp. 325-334, 2002.

INSTITUTO BRASILEIRO DE GEOGRAFIA E ESTATÍSTICA - IBGE. Uma análise das condições de vida da população brasileira. Síntese de Indicadores Sociais. 2007. Rio de Janeiro.. Disponível em: http://www.ibge.gov.br/home/estatistica/populacao/condicaodevida/indicadoresminimos/sinte seindicsociais2007/indic_sociais2007.pdf. Acesso em: Set. 2012.

KLÜCK, M.; GUIMARÃES, J. R.; FERREIRA, J.; PROMPT, C. A. A gestão da qualidade assistencial do Hospital de Clínicas de Porto Alegre: implementação e validação de indicadores. Revista de Administração em Saúde, v. 4, n. 16. Jul./Set. pp. 27-32, 2002.

LAS CASAS, A. L. Qualidade total em serviços: conceitos, exercícios, casos práticos. 5. ed. São Paulo: Atlas, 2006.

LOVELOCK, C.; WRIGHT, L. Serviços: marketing e gestão. Trad. Cid Knipel Moreira. São Paulo: Saraiva, 2001.

MALHOTRA, N. Pesquisa de Marketing: uma orientação aplicada. Trad. Laura Bocco. 4. ed. Porto Alegre: Bookman, 2006.

MCDANIEL, C.; GATES, R. Pesquisa de Marketing. Trad. James F. Suderland Cook. São Paulo: Thomson Learning, 2006.

MELLO, S. C. B.; VIEIRA, R. S. G.; FONSÊCA, F. R. B.; NETO, A. F. S.; GOUVEIA, T. B.; CORDEIRO, A. T. Aplicação do modelo SERVQUAL para a avaliação da qualidade de serviço numa instituição de ensino superior baseado na percepção dos alunos. In: ENCONTRO NACIONAL DE ENGENHARIA DA PRODUÇÃO - Enegep, XXII, 2002, Curitiba. Anais... Disponível em: <http://www.abepro.org.br/biblioteca/ENEGEP2002_ TR26_1034.pdf >. Acesso em: Jun. 2011.

MORETTO NETO, L.; SILVA, J. J. C.; SCHMITT, V. G. H. Introdução à administração hospitalar. Florianópolis: DCA-UFSC, 2007, 74p.

PARASURAMAN, A.; ZEITHAML, V. A.; BERRY, L. L. Um modelo conceitual de qualidade de serviço e suas implicações para a pesquisa no futuro. Revista de Administração de Empresas, v. 46, n. 4, 2006.

SIQUEIRA, A. C. B. Marketing empresarial, industrial e de serviços. São Paulo: Saraiva, 2005. 